\title{
Using the Brink Depth in Discharge Measurement for Inverted Semicircular Open Channels
}

\author{
Riham Zeidan', I. M. H. Rashwan'2, and M. B. A. Khadr ${ }^{3}$ \\ ${ }^{1}$ Master student, Irrigation and Hydraulics Dept., Faculty of Engineering, Tanta University, Egypt \\ ${ }^{2}$ Hydraulic Professor, is with Irrigation and Hydraulic Engineering Department, Faculty of Engineering, Tanta \\ University, Tanta, Egypt, (e-mail:imh_rashwan@yahoo.com, ibrahim.rashwan@f-eng.tanta.edu.eg ). \\ ${ }^{3}$ Associate Professor, is with Irrigation and Hydraulic Engineering Department, Faculty of Engineering, Tanta University, \\ Tanta, Egypt, (e-mail: mosaad.khadr@f_eng.tanta.edu.eg).
}

\begin{abstract}
A free overfall at the end of an open channel provides a simple means for measuring flow discharge. The semicircular open channel plays a significant role in various applications, and it is of interest to calculate its discharge. This paper presents the application of the momentum equation to evaluate the use of a smooth inverted semicircular channel end as devised to calculate the discharge if the end depth is known. The experiments were performed in three separate semicircular inverted channels, with diameters of $15.0 \mathrm{~cm}$, $18.4 \mathrm{~cm}$, and $24.2 \mathrm{~cm}$. The experimental data used to develop equations for calculating the discharge from known end depth. Where the end depth is related to the critical depth, and the end depth ratio value was found to be 0.8102 for a critical depth-diameter ratio of up to 0.40 . On the other hand, the relationships for end depth ratio and non-dimensional discharge are defined. Two empirical equations are generated for the direct and indirect prediction of discharges from known end depth values.
\end{abstract}

Keywords: Brink, End Depth Ratio, Semicircular Open Channel, Overfall,

\section{INTRODUCTION}

A FREE overfall occurs when there is a sudden drop in the bed elevation of a channel causing the flow to separate and form a free nappe. When the approaching flow is subcritical, it is critical to the flow upstream of the free overfall. The pressure distribution is no longer hydrostatic, owing to the vertical acceleration at the brink. The flow depth at the sharp edge is called brink depth or end depth. The ratio of brink end depth $\left(y_{e}\right)$ to normal depth $\left(y_{n}\right)$ is known as the end-depth-ratio $\left(\mathrm{EDR}=y_{e} / y_{n}\right.$ ). The flow ultimately achieves the channel becomes overfall then one consider free overfall (sudden drop) is usually used in open prismatic channels with different shapes as a discharge-measuring device. End depth or brink depth is an easy way to estimate the discharge of all channel types. A possibility to estimate the discharge presented by the ratio of the end depth to the critical flow depth.

Dey [1] measured the end depth of a free overfall in horizontal or slightly sloping inverted semicircular channels. He derived a simplified momentum equation method and contrasted the mathematical model with the experimental results. For a critical diameter ratio of up to 0.86 , he consulted that the end depth varies approximately linearly from 0.72 to 0.74 . The flow upstream of a free overfall from smooth inverted semicircular channels was theoretically studied by Dey [2] based on Boussinesq's assumption of calculating the end-depth ratio (EDR), applying an energy equation. Dey [3] performed both experimental and theoretical studies of a smooth inverted semicircular channel free overfall. He found the expression for the end-depth ratio, based on the momentum method, which removed the need for an empirical pressure coefficient. The flow upstream of a free overfall from smooth inverted semicircular channels formed by Dey et al. [4] is theoretically analyzed for calculating the end-depth ratio (EDR), applying an energy equation based on the Boussinesq assumption. This method removes the need for a pressure coefficient. Raikar et al. [5] presented the application of an artificial neural network (ANN) to evaluate the end-depth-ratio for a smooth inverted semicircular channel in subcritical and supercritical flow. The experimental data used for network testing and validation. Ahmed [6] showed the simulation of the free overfall in an inverted semicircular channel with a sharpcrested weir, the end-depth ratio for the description of the discharge is almost from a single end-depth calculation. $\mathrm{He}$ concluded an average value of 0.713 for this constant. Pal and Goel [7] used the technique of supporting vector machines to predict the end-depth-ratio value and discharge of a free overflow occurring over an inverted smooth semicircular channel and a circular channel with a flat base. Beirami et al. [8] presented based on the free vortex theorem and momentum equation, a theoretical model for predicting the distribution of the pressure head at the end. The pressure coefficient, the end depth ratio, and the flow discharge at the brink of free overfall in channels of various cross-sections with the subcritical flow.

Mohammed et al. [9] showed the relationship between the brink and critical depth, the discharge equations for two models using two free overfall models: straight vertical and skewed end lip, and concluded that the discharge for the skewed lip model was 13 percent higher than the straight vertical model. Nabavi [10] researched a theoretical model for measuring the distribution of the pressure head at inverted semicircular channels. Applied momentum equation, then obtains the ratio of end depth. Similar to critical depth for design purposes, charts were constructed to predict flow discharge when the end-depth value for a critical depth-diameter ratio of up to 0.4 is defined as the value of (0.7). Nabavi et al. [11] displayed a theoretical model for measuring the flow of horizontal or mildly sloping inverted semicircular channels by the end depth method. A momentum method was applied to provide an expression for EDR, the value was found to be 0.7 for the ratio of critical depth diameter up to 0.4. They also gave expression on discharge. Navabi et al. [12] performed a theoretical model based on the free vortex theorem, which is capable of predicting the distribution of the pressure head 
at the brink of free overfall in open channels. By applying the momentum equations to obtain the end-depth-ratio from which the discharge can be calculated, in the adverse sloping or rough channel. The theory is successfully applied to flat-based circular and U-shaped channels. Swetapadma et al. 2014 [13] offered a brink-depth analysis by different investigators for a clear understanding of all of its aspects. They used the key theories, theory, equations, and engaged modeling techniques along with the findings that were discussed in brief. This analysis provided for different channel shapes under the categories below. Lamri [14] examined the uniform flow in a semicircular conduit. He calculates the conduit radius by using calculation steps that he solves the problem by evaluating the linear dimension, forgiven discharge data passes through the pipe. The channel architecture depends on the Rough Reference Model Theory. Gupta et al. [15] based on applying the momentum equation with some assumptions, and at the end part, a few based on energy consideration and water surface profile. The general mathematical model is derived from the use of momentum, discharge, and Froude number equations for open channels with horizontal, moderate, and adverse slopes. Prior experimental data calibrate a proposed mathematical model. To measure the relationship between EDR and EDD for exponential channel cross-sections in supercritical flow regimes, Abrari et al. [16] using the momentum equation based on free vortex theory. Abrari et al. [17] concluded that the end depth of a free overfall gives a clear indicator of how to discharge should be measured. The scientific note discusses two theoretical methods for end-depth computation (EDR) and end-depth discharge (EDD) relationships based on the momentum and energy equations for inverted semicircular channel cross-sections. With the use of two theoretical approaches, the flow upstream of a free overfall has been theoretically investigated to compute the end depth ratio and the end depth discharge of an inverted semicircular channel crosssection on a steep slope. Two empirical equations are generated mathematically for the direct prediction of discharges from known end depth values.

Thus, this study presents the evaluation efficiency for brink as a discharge measurement device for a partially filled semicircular open channel. The main target of the present study derived relationships between various variables of the semicircular channel from laboratory data in new forms of equation and figures.

\section{METHODOLOGY.}

A sudden drop at end of the open channel bed as shown in Fig. 1. characterizes a free-fall over a channel. It also shows a uniform flow followed by gradually varying flow and rapidly varying flow, at the end of which brink depth occurs just at the free overfall.

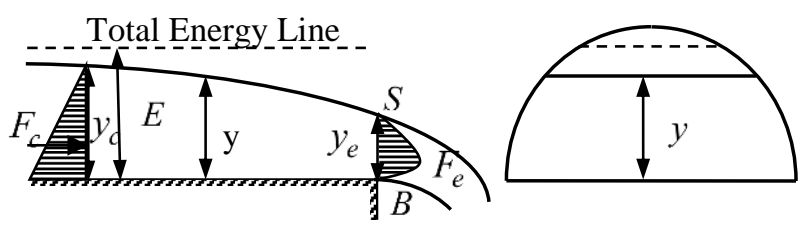

Fig. 1. Free overfall (Brink)
Flow over a free fall splits at the sharp edge or drop in the form of a nappe and leads to rapidly varying flow with an appreciable curvature of streamlines creating a nohydrostatic distribution of pressure. The flow depth at the brink is, therefore, lower than the critical flow depth and is called end depth or brink depth. This type of drop occurs in the city drainage system and hydraulic drop-style structures used in irrigation engineerings such as notches and fallings. The pressure at the top and bottom ends ( $\mathrm{S}$ and $\mathrm{B}$ in Figure (1)) of the dropping nappe at the brink or end of a channel is atmospheric and varies almost parabolic.

Taking into account all the effective forces parallel to the channel bed, the momentum equation may be written as follows:

$$
\rho Q\left(\beta_{e} V_{e}-\beta_{c} V_{c}\right)=F_{c}-F_{e}-W \sin \phi-F_{f}
$$

Where $\rho$ is the water density, $Q$ is the discharge, $\beta_{c}$ is the momentum coefficient at section $1, \beta_{e}$ is the momentum coefficient at section $2, V_{c}$ is the mean flow velocity at section $1, V_{e}$ is the mean flow velocity at section $2, F_{c}$ and $F_{e}$ are the resultants of pressures acting on the two sections, $W$ is the weight of water enclosed between the two sections, $\phi$ is the bed slope angle and $F_{f}$ is the total external force of friction and resistance acting along the surface of contact between water and the channel bed

For the horizontal channel, the horizontal component of the weight of the water enclosed between the two sections equals zero, $W=0$.Also, the length between the two sections is so small that the external force of friction on the channel perimeter is negligible, $F_{f}=0$. The velocity distribution is assumed to be uniform; then $B_{c} \cong \beta_{e} \cong 1.0$. With using the continuity equation, Eq. (2) becomes

$$
\frac{\gamma Q^{2}}{g A_{c}}+F_{c}=\frac{\gamma Q^{2}}{g A_{e}}+F_{e}
$$

Where $A_{c}$ is the cross-sectional area of critical flow and $A_{e}$ is the cross-sectional area of end flow, While $\rho=\gamma / g$ and $V=Q / A$ where $\gamma$ is the unit weight of water, $g$ is the gravitational acceleration, and $A$ is the cross-sectional area of flow

Froude number in open channels is expressed as

$$
F_{r}^{2}=\frac{V^{2}}{g y_{h}}=\frac{Q^{2} T}{g A^{3}}
$$

Where $F_{r}^{2}$ is Froude number, $y_{h}$ is hydraulic mean depth ( $A / T$ ), and $T$ is the top width

For critical flow at the upstream section, $F_{r c}{ }^{2}=1$

$$
\frac{Q^{2}}{g}=\frac{A_{c}^{3}}{T_{c}}
$$

Put Eq. (5) in Eq. (3) gave

$\gamma A_{c}^{2}+F_{c}=\frac{\gamma A_{c}^{3}}{A_{e}}+F_{e}$

\section{A. Upstream Hydrostatic Force}

The upstream hydrostatic force

$$
F_{c}=\gamma A_{c} \bar{y}_{c}
$$


Where $\bar{y}_{c}$ is the centric depth of the segment area from the free surface of the water

\section{B. Downstream Hydrostatic Force}

The upstream hydrostatic force as Beirami et al [8]

$$
F_{e}=K_{e}\left(\gamma A_{e} \bar{y}_{e}\right)
$$

Where $K_{e}$ is the assumed coefficient of pressure distribution

\section{The Brink Equation}

Introducing Eq. (8) and Eq. (7) in Eq. (6) and dividing both sides by $\gamma D^{3}$ one gets

$$
y_{h c^{*}}+\bar{y}_{c^{*}}=\frac{y_{h c^{*}}}{A_{r}}+K_{e}\left(A_{r} \bar{y}_{e^{*}}\right) \ldots
$$

Where $y_{h c^{*}}$ is dimensionless hydraulic mean depth $\left(y_{h c} / D^{3}\right), D$ is the diameter of channel, $\bar{y}_{c^{*}}$ is the dimensionless centric depth of the segment area from the free surface of the water for critical section $\left(\bar{y}_{c} / D\right), A_{r}$ is the relative area $\left(A_{e} / A_{c}\right)$, and $\bar{y}_{e}$ is the dimensionless centric depth of the segment area from the free surface of the water for the end $\operatorname{section}\left(\bar{y}_{e} / D\right)$

\section{Geometric Properties of Semicircular Channel}

The geometric properties of the inverted segment of the semicircular can be easily determined. The exact dimensionless cross-sectional area, $A_{*}$, of the partially filled inverted semicircular channel can be expressed in dimensionless form as:

$$
A_{*}=\frac{A}{D^{2}}=\frac{\theta-\sin \theta-\pi}{8} \text {. }
$$

Where $A_{*}$ is the dimensionless water area, $A$ is the crosssectional area of the partially filled inverted semicircular channel, $\theta$ is the central angle of the cross-sectional area of the partially filled inverted semicircular channel, and $\pi$ is constant equals to 3.14 as shown in Fig. 2 .

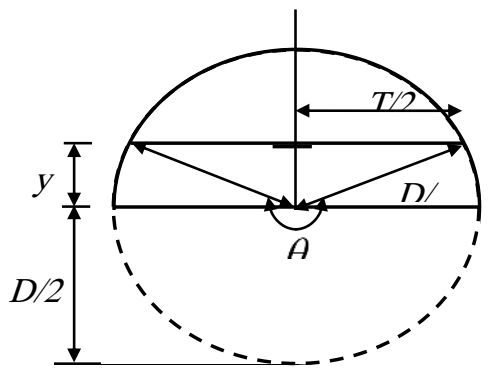

Fig. 2. Geometric properties

The dimensionless top width is maybe written as;

$$
T_{*}=2 \sqrt{0.25-Y^{2}}
$$

Where $T_{*}$ is dimensionless top width $(T / D)$, and $Y$ is dimensionless water depth $(y / D)$

\section{E. Centric depth of the semicircular section}

The dimensionless relative centric depth of the segment area of a circular cross-section from the free surface of the water may be written as follows:
$\overline{Y_{s}}=\frac{2}{3}\left(\frac{\sin ^{3}(\theta / 2)}{\theta-\sin \theta}\right)-\frac{\cos (\theta / 2)}{2}$

Where $\bar{Y}_{s}$ is the centric depth of water area of a segment of a circle, $\left(\bar{Y}_{s}=\bar{y}_{s} / D\right)$

For central angle of the cross-sectional area of the partially filled inverted semicircular channel as shown in Figure (2).

$$
\cos (\theta / 2)=\frac{D-2 y}{D}=1-2 Y
$$

And

$$
\sin (\theta / 2)=\frac{2 \sqrt{D y-2 y^{2}}}{D}=2 \sqrt{Y-Y^{2}}
$$

Herein, the dimensionless centric depth of the semicircular channel becomes

$$
\bar{Y}=\frac{2}{3 A_{s}}\left(Y-Y^{2}\right)^{3 / 2}-\left(\frac{1}{2}-Y\right)-\frac{1}{A_{s}}\left(\frac{\pi}{8} Y-0.11292\right) .
$$

Where $\bar{Y}$ is the dimensionless centric depth of water area of a segment of inverted semicircular cross-sectional $\bar{Y}=\bar{y} / d$

\section{EXPERIMENTAL WORK}

The flume used with dimensions of $14.25 \mathrm{~m}$ long, $1.00 \mathrm{~m}$ wide, and $1.00 \mathrm{~m}$ in height. It was divided into three sections. The first one was used to remove eddies and to give a uniform flow condition. The second part of the horizontal slope flume has an inverted semicircular flume of length $(6.00 \mathrm{~m})$ with internal variable diameters (D) equals to $15.0,18.4$, and $24.2 \mathrm{~cm}$. Plate (1). The water depth in the inverted semicircular flume can be read using piezometers. Third-part escapes with a rectangular notch to measure the discharge. A laboratory of 100 experimental runs in the inverted semicircular flumes with different diameters for different discharges was conducted.

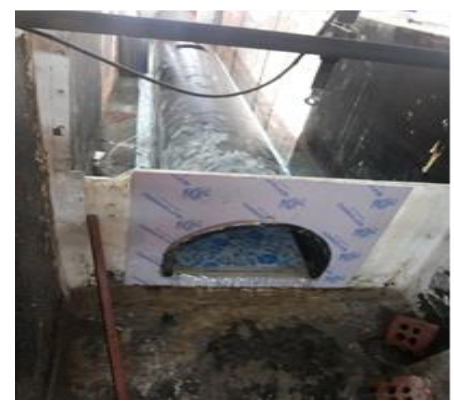

Plate I Inverted Semicircular Horizontal Flume

The main purposes were to deduce equations for discharge with end depth for inverted semicircular open channels. The water depths at the end of the flumes and calculated discharges are presented in TABLE I.

\section{RESULTS AND ANALYSIS}

A free overfall at the end of an open channel provides a simple means for measuring flow discharge. This paper presents the application of the momentum equation to evaluate the end-depth-ratio for a smooth inverted semicircular channel. The experimental data used to develop equations for calculating the discharge from known end depth. 
TABLE I EXPERIMENTAL DATA COLLECTED

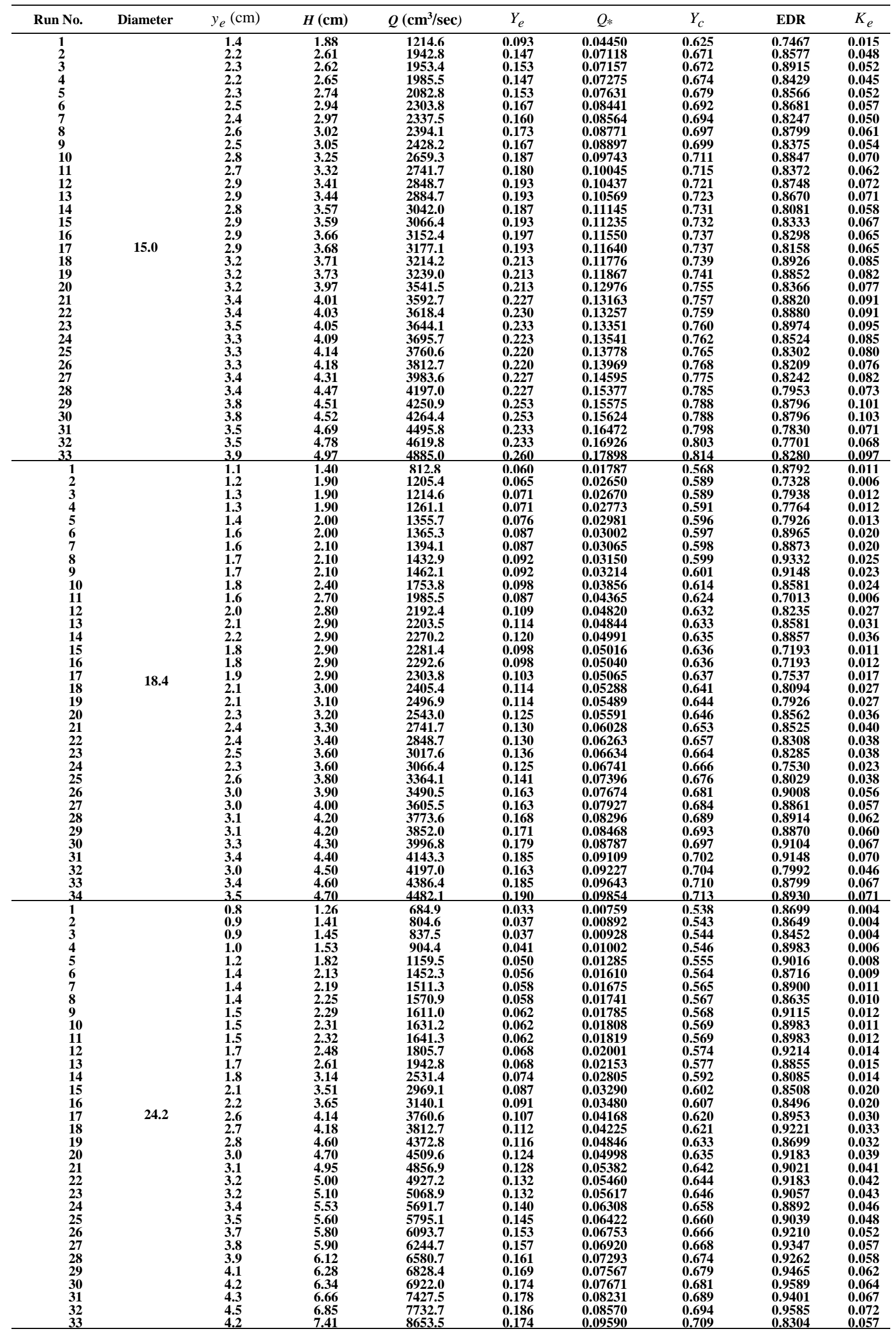


The discharge can be easily measured if the end depth is known, and a connection exists between the end depth and the critical flow depth, Fig. 1. The estimation of relative critical depth, the dimensionless discharge $Q_{*}=\sqrt{Q^{2} / g D^{5}}$ , the end depth ratio, and the pressure coefficient are presented in TABLE I.

The present study provides a simplified approach to evaluate the end depth of a free overfall in semicircular channels recognizing that few attempts have been made in this form of the section to examine the free overfall. Checked with different discharges and three pipe diameters $15 \mathrm{~cm}, 18.4 \mathrm{~cm}$, and $24.2 \mathrm{~cm}$, it can be developed discharge with end depth equation.

\section{A. Direct estimate of the discharge}

Fig. 3 can be used to estimate directly the discharge from measured end-depth. The relationship between the dimensionless measured discharge, $Q_{*}$ and the dimensionless end water depth, $Y_{e}$ can be written as follows using the least square techniques,

$$
Q_{*}=1.2393 Y_{e}^{1.4898} \ldots \ldots \ldots . . R^{2}=0.9821
$$

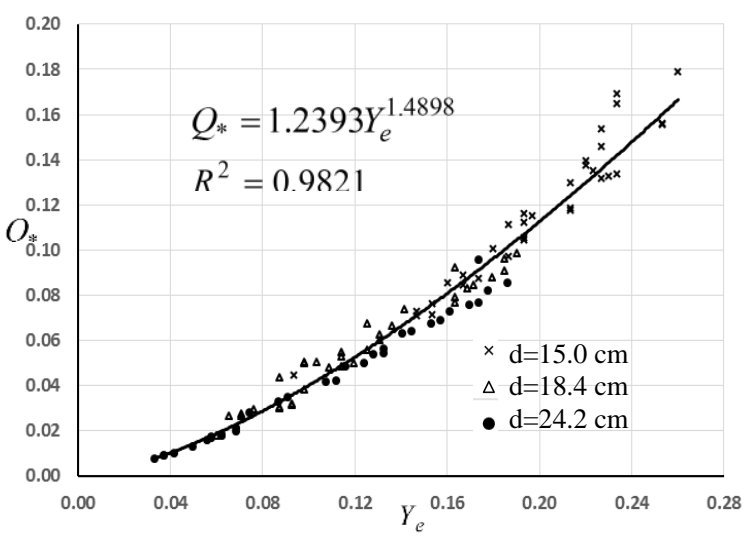

Fig. 3. Variation of relative end depth, $Y_{e}$ with dimensionless discharge

$$
Q_{*} \text { for different pipe diameters }
$$

Eq. (16) generated mathematically for the direct prediction of discharges from known end depth values. Checked with different discharges and three pipe diameters, it can be cleared that the radius not affected the relationship under consideration.

\section{B. An indirect estimate of the discharge}

The relationship between critical water depths $Y_{c}$ is shown in Fig. 4. The relationship is a straight line closely correlates with accuracy to the experimental results.

The relationship between the dimensionless critical depth, $Y_{c}$ and the dimensionless end water depth, $Y_{e}$ can be written as follows using the least square techniques,

$$
Y_{c}=1.1597 Y_{e}+0.001 \ldots \ldots \ldots . R^{2}=0.9768 \ldots
$$

For a given end depth $Y_{e}, Y_{c}$ is computed from Eq. (17). Then, the discharge is solved numerically from Eq. (5) or by using the following relationship, Fig. 5 .
The relationship between the dimensionless measured discharge, $Q_{*}$ and the dimensionless critical water depth, $Y_{c}$ can be written as follows using the least square techniques, $Q_{*}=1.0335 Y_{c}^{3.0138}$ $R^{2}=1.0000$

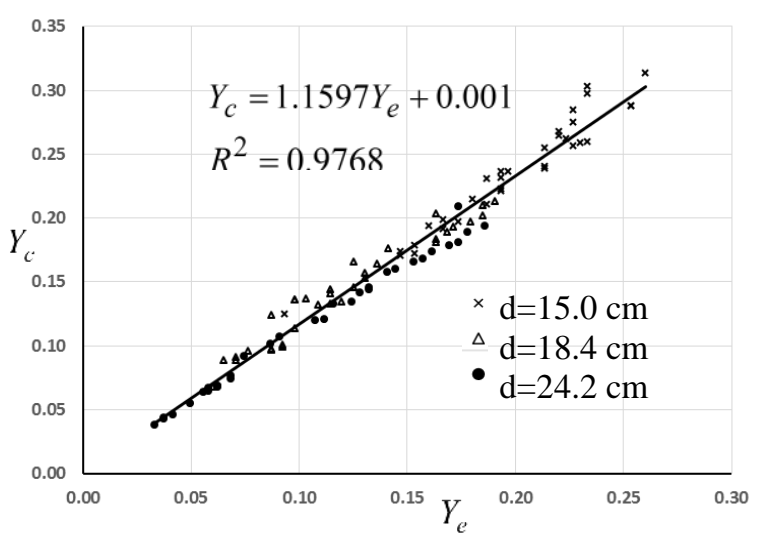

Fig. 4. Variations of relative critical depth $Y_{c}$ with relative end depth $Y_{e}$

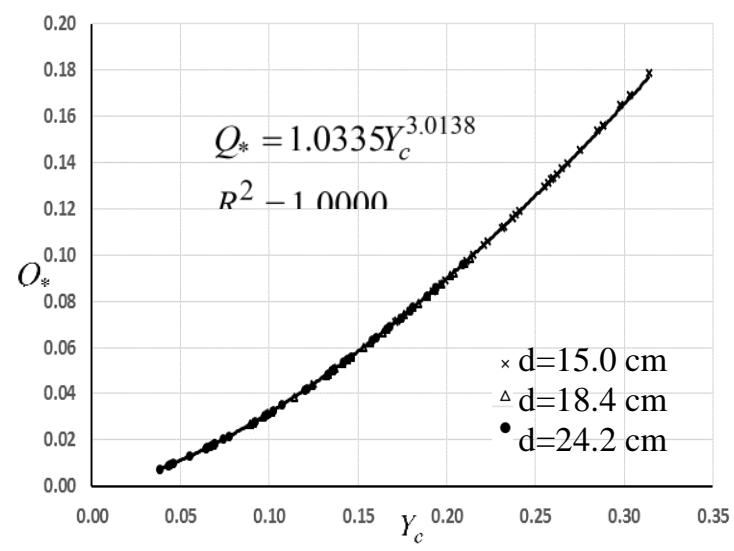

Fig. 5. Variations of $Q_{*}$ with $Y_{c}$

Again for a given end depth $Y_{e}, Y_{c}$ is computed from Eq. (17). Then, the discharge is solved numerically from Eq. (18). For a given end depth $Y_{e}, Y_{c}$ is computed from Eq. (9). Then, the discharge is solved numerically from Eq. (5).

\section{An estimate of the discharge using Momentum Equation}

As the value $K_{e}$ for flow over free overfall is not available as yet, Eq. (9) is required to be calibrated extensively $K_{e}$. The experimental data were used to calibrate Eq. (9), making $K_{e}$ a free parameter. For this purpose, the experimental data for discharge and different end depths were used as source data. The variation of $K_{e}$ with $Y_{e}$ is shown in Fig. 6, where $K_{e}$ decreases with increase $Y_{e}$.

The relationship between the pressure distribution coefficient, $K_{e}$ and the dimensionless end water depth, $Y_{e}$ can be written as follows using the least square techniques, $K_{e}=0.452 Y_{e}-0.0191$ $R^{2}=0.9580$

Again for a given end depth $Y_{e}, K_{e}$ is computed from Eq. (19), then, the critical water depth is solved numerically 
from Eq. (9). For the computed relative critical depth $Y_{c}$, the discharge is solved numerically from Eq. (5).

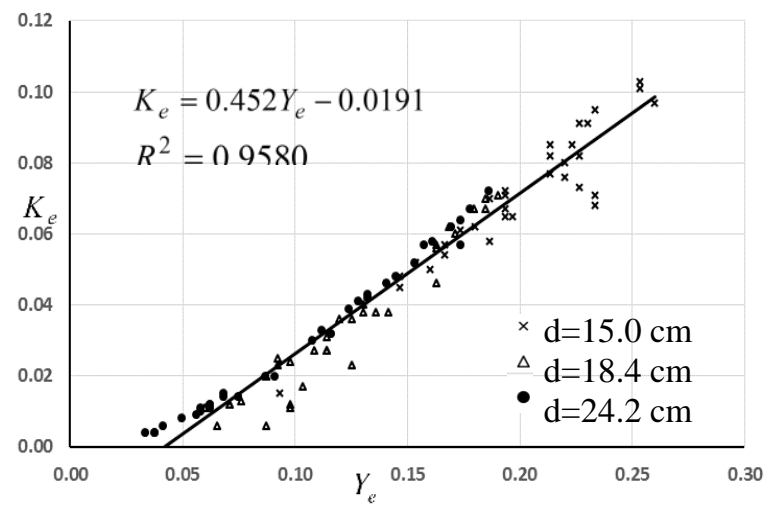

Fig. 6. Variations of pressure distribution coefficient $K_{e}$ with relative end depth $Y_{e}$

\section{End depth ratio (EDR)}

The end depth ratio $Y_{e^{*}}$ is the relation between end depth and approaching critical water depth. According to the present experiment, the end depth ratios vary almost from 0.7013 up to 0.9589 . The variations $Y_{e^{*}}$ are presented in Fig. 7., with a mean value of 0.8201 for different values of channel diameters.

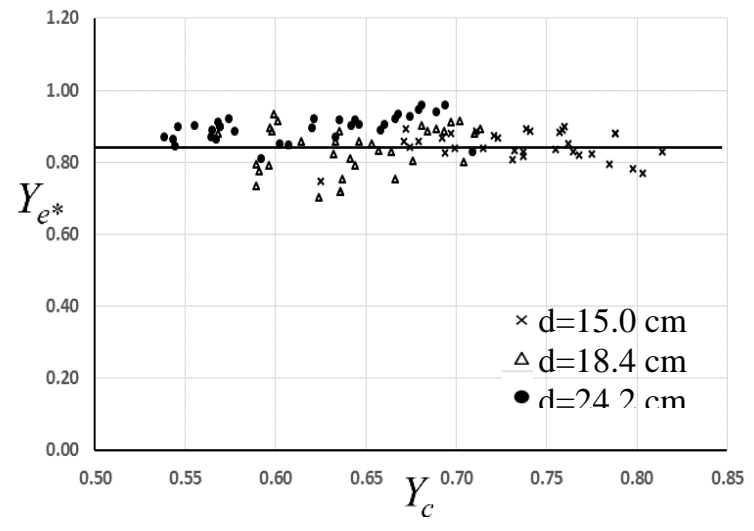

Fig. 7. Variations of end depth ratios $Y_{e^{*}}$ with relative critical water depth $Y_{c}$

\section{CONCLUSIONS}

The flow upstream of a free overfall was investigated to compute the discharge of an inverted semicircular channel cross-section on a horizontal slope. The present study presents the application of the momentum equation to evaluate the use of a smooth inverted semicircular channel end as devised to calculate the discharge if the end depth is known. Also, the experimental data used to develop equations for calculating the discharge from known end depth. The equations are generated for the direct and indirect prediction of discharges from known end depth values.

\section{A. Direct estimate of the discharge}

Eq. (16) can be used mathematically for the direct prediction of discharges from known end depth values. The equation yielded a highly satisfactory agreement with experimental data sets.

\section{B. An indirect estimate of the discharge}

- For a given end depth $Y_{e}, Y_{c}$ is computed from Eq. (17). Then, the discharge is solved numerically from Eq. (18). Another model for indirect solution for a given end depth $Y_{e}, Y_{c}$ is computed from Eq. (9). Then, the discharge is solved numerically from Eq. (5).

- Checked with different discharges and three pipe diameters $15 \mathrm{~cm}, 18.4 \mathrm{~cm}$, and $24.2 \mathrm{~cm}$, it can be cleared that the radius not affected the relationship between discharge and end depth.

- The end depth is related to the critical depth, and the end depth ratio value was found to be 0.8102 for a critical depth-diameter ratio of up to 0.40 .

\section{REFERENCES}

[1] Dey, S., Flow measurement by the end-depth method in inverted semicircular channels, Flow Measurement and Instrumentation 12 (4) (2001) 253-258.

[2] Dey, S., "Free overall in circular channels with flat base: a method of open channel flow measurement." Flow Measurement and Instrumentation, 10.1016/S0955-5986(02)00061-4, 209-221 . Online publication date: 1-Dec-2002.

[3] Dey, S., Kumar, D. and Singh, D., End depth in inverted semicircular channels: experimental and theoretical studies. Nordic Hydrology, 35 (1): 73-79. 2003.

[4] Dey, S., "Free Over Fall in Inverted Semicircular Channels", Journal of Hydraulic Engineering, ASCE, 129:438-447, 2003.

[5] Rikar, R. V., Kumar, D. N., and Dey, S, "End depth computation in inverted semicircular channels using ANNs." Flow. Meas. Instrum., 15(5), 285-293, 2004.

[6] Ahmed Z., "Flow Measurement Using Free Overfall in inverted Semi-Circular Channel", Flow Measurement and Instrumentation, 16(10), 21-26, 2005.

[7] Pal M. and Goel A., "Prediction of The End-Depth Ratio and Discharge in Semicircular and Circular Sloped Channels Using Supported Vector Machine, Flow Measurement, and Instrumentation, 17(3), 49-57, 2006.

[8] Beirami M.K., Nabavi S.V., and Chamani M.R. "Free overfall in channels with different cross-sections and sub-critical flow", Iranian Journal of Science and Technology, Vol. 30, No. B1, 2006, pp. 97-105.

[9] Mohammed, A.Y., Moayed, S.K. and Mohammad, M.Y., Variation of water depth on normal and skewed broad crested weirs, Tikret Univ. Journal of Engineering, Irrigation and Drainage Engineering, ASCE, 125:40-4, 2007.

[10] Nabavi, Seyed Vahid, End depth as measuring device in inverted semicircular channels. In: 3rd IASME/WSEAS int. conf. on water resources, hydraulics, and hydrology (WHH '08). University of Cambridge, UK; 2008. p. 23-5.

[11] Nabavi S. V., Beirami M. K., Sterling M.” Flow Metering By End-Depth Method in Inverted Semicircular Channels" Proceedings of the 4th IASME/WSEAS Int. Conference on Water Resources, Hydraulics, and Hydrology (Whh'09), 2009.

[12] Nabavi, S. V., Beirami, M. K., Chamani, M. R., and Sterling, M. (2011). "Free over falls in flat-based circular and U-shaped channels, Flow. Meas. Instrum. , 22(1), 17 -24, 2011.

[13] Swetapadma Sonali, Mittal, S. K., and Choudhary, M. K., Brink depth at free overfall-A review- Brink Depth at Free Overfall - A Review October, International Journal of Engineering Research V No.3, Issue No.10, pp: 594-601, 2014.

[14] Lamri Ahmed Amine," Dimensioning of the Semicircular Open Conduit", 2014.

[15] Gupta, V., Tiwari, H. L., and Mittal S. K., Brink depth at free overfall in Open Channel Analysis: A review, International Journal of Scientific Research and Development, Volume: 4, Issue: 3, 2016.

[16] Abrari, E. Ergil, M., and Beirami, M. K., "Free overfall in exponential channel cross-sections based on free vortex theory in 
supercritical flow regimes.” Flow. Meas. Instrum. , 50, $269-279$, 2016.

[17] Abrari, E., Ergil, M. and Beirami, M. K., Direct Prediction of Discharge at Supercritical Flow Regime Based on Brink Depth for Inverted Semicircular Channels, Journal of Irrigation and Drainage Engineering, vol. 143 issue 9, September 2017. 